\title{
The relative importance of specific immunity in protecting against leprosy
}

\author{
A BRYCESON \\ Clinical Research Centre, P O Box 20778 Nairobi
}

The title of this paper contains four words, leprosy, immunity, protection and relative, of variable meaning which need working definitions, and will be considered in turn.

\section{Leprosy}

The clinical disease, leprosy, is important because it causes disability and because it maintains the source of infection in the community and thus permits further disease and disability. Subclinical infection is important too: it may induce immunity and protect against subsequent infection and so prevent disease, or it may maintain a potential reservoir of infection in the community which is hard to detect. Both these forms of leprosy need to be considered.

\section{Specific Immunity}

This is taken to mean the host response, specific to M. Leprae that follows exposure to M.leprae or its antigens. As so little is known about protective mechanism in leprosy and their specificity, it will be necessary to review some of the phenomena that have been observed during and after infection with M.leprae, and to discuss their possible significance in several situations where protection against infection might be immunologically mediated.

\section{CELL MEDIATED IMMUNITY IN ESTABLISHED LEPROSY}

The immune response in leprosy may be measured in several ways. In overt disease clinical assessment and histological classification supported by the bacterial index and the lepromin test provide an adequate measure of cell mediated immunity (CMI), the Ridley-Jopling scale. But these methods are of no value in epidemiological studies.

Godal (1) and his coworkers made three major contributions when they developed and applied in vitro tests of CMI to the study of leprosy. They confirmed the immunological basis of the RidleyJopling scale, leaving it as a reference standard, they provided a means of detecting subclinical infection with M.leprae and, in so doing, they provided the first useful immunological tool for epidemiological use. 
The striking immunological feature of established disease is the presence or absence of CMI and its relationship with resistance to the spread of M.leprae. It has been shown that CMI makes three main contributions to the disease. By damaging nerves it is the main cause of disability, it provides a means of eliminating M.leprae (2) and by failing it permits the spread of M.leprae in the patient and the community. In the 1960s, Godal and his colleagues described the total absence of in vitro CMI responses to whole M.leprae in patients with lepromatous leprosy and concluded that there was an absolute deficiency of specifically sensitised lymphocytes (3).

Recently, suppressor cells have been demonstrated by different techniques and in different situations in leprosy. Nath (4) found that lymphocytes from tuberculoid patients suppressed the lymphoproliferative response to HLA matched allogenic cells to Concanavalin $A$ or to lepromin. Lymphocytes from lepromatous patients did not suppress in this situation. She also found that lepromin suppressed the response to Con A of lymphocytes from $80 \%$ of tuberculoid patients, but only 35\% of lepromatous patients. Suppressor activity in tuberculoid patients may simply reflect normal regulatory mechanisms, but it might also be induced by M.leprae and represent a mechanism for bacillary persistence, relapse and downgrading as Stoner has suggested (5). Mehra (6) reported different results, possibly due to the different culture condition relating to the timing of mitogen addition. She found that lepromin caused a marked suppression of Con A responses by blood mononuclear cells from lepromatous, but not tuberculoid patients. She identified suppressor activity in two types of cells, adherent cells and $T_{\gamma}$ cells(7). Finally A-M Bach reported that patients with errythema nodosum leprosum (ENL) had a reduction of circulating lymphocytes bearing the surface marker OKT8. These cells normally have suppressor activity. Patients with uncomplicated lepromatous leprosy and or tuberculoid leprosy had normal numbers of cells (paper read at the Conference on Immunological Aspects of Leprosy. Tuberculosis and Leishmaniasis, Addis Ababa, October 27-30, 1980). These results suggest that there are several situations in which different suppressor cells play different roles in leprosy; some of these roles may normally be regulatory, others may be pathological. In lepromatous leprosy specifically sensitised lymphocytes are present. not absent, but they are suppressors, not helpers and may possibly provide a mechanism for the total suppression of CMI in lepromatous disease. In certain circumstances, M.leprae can suppress lymphocyte responses to mitogens (6) or PPD (5) and at high concentrations may be lymphocytotoxic ( 8 ).

None of these studies, however, yet answers the question: is the immune suppression genetically determined, or is it induced by M.leprae or some other mycobacteria; or, as seems likely, are genetic and acquired determinants both important, each dominating a particular situation? 


\section{SPECIFICITY OF TESTS OF CMI IN LEPROSY}

Delayed hypersensitivity skin tests with Dharmendra lepromin, read at 48-72 hours (Fernandez tests) have not as a rule clearly distinguished infection with M. leprae from other mycobacteria. Although specificity appears to be better using the soluble protein extract of armadillo-derived leprosy bacilli( leprosin), firm conclusions must await definitive extraction and preparation procedures (9).

LTT responses to whole M.leprae and BCG show very clear separation between leprosy and tuberculosis, especially when used epidemiologically rather than clinically (10). The lack of complete distinction of specificity that Closs reported among healthy Norwegians after BCG inoculation may have been due to his use of freeze thawed bacilli (1l). The ability of BCG inoculation to precipitate type 1 lepra reactions in borderline disease, suggests that cross reactivity to antigens of disintegrated bacilli is of clinical importance (12). The specificity of the defective CMI in lepromatous leprosy is strikingly and curiously specific: LTT responses are absent to wholeleprae but normal to BCG (3). This observation should contain a clue to the mechanism of suppression: Does M.leprae present itself to the host as a single antigenic 'package' or is the defect restricted to a particular surface antigen, or antigens? Lymphocyte responses in vitro have been studied with regard to one purified antigen, antigen 7 M.fleprae (14), but there are too few data to discuss the significance of CMI responses to individual antigens.

\section{HUMORAL RESPONSES IN ESTABLISHED LEPROSY}

The recent development of sensitive tests to detect antibodies specific to M.leprae crossed immuno-electrophoresis by Axelson (15), radio-immunoassay by Harboe (16) and immunofluorescence by Abe $(17,18)$ have proved remarkably sensitive and specific.

These tests have made three, at least, major contributions to the study of leprosy. Their application to the spectrum of leprosy has led to the rejection of the notion that only lepromatous patients produce antibody, although classical precipitating antibody is restricted to patients with $L L$ and $B L$ disease (19), and seems to correlate with liability, to type 2 reactions. Secondly they provide another sensitive epidemiological tool and thirdly, crossed immuno-electrophoresis has helped in the antigenic analysis of M. Leprae.

The demonstration of antibodies in patients across the spectrum raises two questions: what are they directed against and what might be their biological functions? Harboe and Closs and their colleagues $(13,20)$ found that they were directed against all of the seven antigens originally detected in M.leprae by crossed immunoelectrophoresis. There are two curious results of these studies: the paucity of antigens in M.leprae when compared with other myco- 
bacteria, and the lack of antibody specificity. More sensitive techniques showed the presence of up to 20 antigens, but the relative difficulty in demonstrating them remains unexplained. Perhaps certain M.leprae antigens are in some way less immunogenic than usual or even tolerogenic. The concept of tolerogens has been considered in relation to certain species of Leishmania, which are capable of causing diffuse cutaneous leishmaniasis, a condition analagous to lepromatous leprosy, in a small proportion of infected individuals (22). All of Harboes 7 antigens were shared with a wide range of other mycobacteria although the diffusion patterns suggested the possibility of specific determinants on common antigens. More recently there have been reports suggesting the identification of specific antigens (23). Specific serological activity on the other hand has been demonstrated consistently by Harboe and Abe using cross absorbtion tecnhiques, and although such techniques are valuable for epidemiological purposes, the study of responses to characterised specific antigens may be necessary for a fuller understanding of the immune defect in lepromatous leprosy. Antibody activity has been demonstrated against both cell wall and cytoplasmic components, but the biological function of these antigens antibodies remains unknown. Antibody detected by RIA to cell wall antigen 7, appears to act, as a marker of activity in lepromatous and in borderline disease (24), but there is nothing to suggest it has a suppressive function. Conversely no protective role for specific antibody has been demonstrated yet, but its presence as a rule in tuberculoid and subclinical cases should make us reconsider the notion that CMI alone protects against leprosy. Lurie (25) showed, not only that macrophages provided the essential protective mechanism in rabbits immune to tuberculosis, but also that serum factors inhibited the growth of M.tuberculosis implanted in agar into these animals. Poulter's recent studies of the guinea pig model of cutaneous leishmaniasis, have shown that antibody appearing at a particular time after recovery from Leishmania enriettii infection confers passive immunity to naive animals (26). Previously CMI alone was thought to be protective.

\section{IMMUNE RESPONSES IN SUBCLINICAL LEPROSY}

These responses, which are of the greatest interest to this meeting, will be discussed by others in detail, but a brief summary is necessary here to understand the background against which we must consider the role of specific protection. Two papers have radically altered our concepts of the infectivity of leprosy and of the immune response to infection, as opposed to disease. Godal and Negassie (10), using the LTT to M.leprae in three separate comparisons, showed that $29 \%$ of newcomers to an endemic area developed positivity, presumably due to infection, after one or more years residence in an endemic area, that occupational contact increased positivity to $53 \%$, and that positivity increased with the degree of contact: 
$88 \%$ of (only 17) workers in the out-patient department at Addis Ababa leprosarium being positive. Abe's (18) contribution is increased by the complete specificity of the FAT for M.leprae, after absorbtion of the test sera with BCG, M.vaccae, and on occasion M.smegmatis. He showed that 92\% (of 62) household contacts of leprosy patients have antibody specific to M.leprae. This is about double the percentage Godal found with the LTT in Ethiopia, where leprosy is more highly endemic. Abe found the highest titres in children under 4 years, in children rather than siblings and in contacts of lepromatous rather than tuberculoid patients.

In attempting to interpret these results certain caveats must be expressed. 1. None of these figures has been related to prevalence or incidence figures for the leprosy. Abe's statement that the seropositive rate among schoolchildren with suspicious lesions was 200 times the expected incidence of leprosy in that area does not provide the data to work out a relationship between infection and prevalence and incidence of disease. 2. It is not clear whether all people infected with M. leprae will develop an immune response measurable by one of these two, or indeed any other, tests. 3. There has been no comparison between the two tests for sensitivity and correlation in the same community. The FAT seems generally more sensitive, yet where transmission is intense, the gap between the two tests appears to narrow. Abe found an incomplete correlation between seropositivity and Mitsuda positivity in a small number of contacts, but lepromin testing is not the same as the LTT. Nevertheless certain tentative conclusions may be drawn, if only to provide testable hypotheses. 1. In the endemic situation leprosy is a highly infectious disease with a low attack rate. Transmission among contacts does not diminish even when prevalence and incidence are low as in Okinawa. In households, 90\% of child contacts are infected by the age of five. 2. Infection is usually followed by an immune response. 3. Infection increases with closeness of contact, and thus presumably with intensity of transmission in a low endemic area (Japan) equally as in a high endemic area (Ethiopia). This view is supported by Lara's figures for leprosy in child contacts in Culion (27) and by Melsom's demonstration that M.leprae specific antibodies do not decline as expected in neonates born to infectious mothers (28). 4. Intensity of transmission is associated with greater bacterial multiplication in the contact, as evidenced by higher antibody titres. The significance of these conclusions will become clear in considering the question of protection.

What is the evidence that specific immunity protects against leprosy?

The chain of events from organism to disease starts with the source of infection. I find it helpful to think of this as the lepromatous nose, showering clouds of bacilli laden droplets into the atmosphere, in the house, the market, the bus, the school, the pub, the factory, the cinema, the place of worship. One lepromatous patient 
is likely to infect tens, hundreds or thousands of people before the disease becomes apparent and is brought under control (29). There are then five points in the chain at which the immune response might conceivably prevent the development of disease.

1. PROTECTION AGAINST THE ESTABLISHMENT OF INFECTION BY M.LEPRAE AFTER EXPOSURE: There are two possible situations: congenital immunity and immunity acquired from previous natural or artificial infection with other mycobacteria. The evidence for the latter is slender and largely hypothetical but I will allude to it briefly later in considering the term 'relative'. There is however a real possibility that congenital immunity exists. Lara (27) analysed the leprosy rate among children in Culion colony continuously in contact with infectious parents. Of those that developed leprosy, 95\% did so within 3 years, 66\% within 2 years, but only $0.5 \%$ within 1 year. Although the incubation period of leprosy is imperfectly known, periods of under one year are recorded and in such a susceptible group so heavily exposed to infection, one might expect a higher rate before one year. Possible mechanisms for congenital protection exist, transplacental passage of antibody and of specifically primed $T$ cells, have been demonstrated $(28,30)$ and breast milk might contain specific IgA antibodies. IgA is increased in lepromatous patients and in cord blood from neonates of lepromatous mothers, but the presence of antibody in secretions has not been looked for. If such passive immunity exists it would appear normally not to offer lasting protection (27). It is however conceivable that chemotherapy during this early period might permit immunity to be acquired.

2. PROTECTION AGAINST THE DEVELOPMENT OF DISEASE AFTER INFECTION HAS BEEN ESTABLISHED: The age-old observation that recovery from disease at the tuberculoid end of the spectrum is followed by life long immunity makes the case that immunity follows infection. But this immunity follows disease, not subclinical infection, which may be a different situation.

In studies of inbred mice, infected with Leishmania donovani, Bradley (31) showed that innate resistance or susceptibility to the establishment of infection was genetically determined and was not immunologically mediated. It is not known whether such innately resistant mice developed any immunological evidence of infection. One must be cautious therefore in assuming that in man the absence of an immune response to M.leprae indicates no infection: it could indicate non-take. Conversely immune conversion may not necessarily indicate that the infection became established, or took. Furthermore, since lepromin testing with heatkilled M.leprae can induce Mitsuda positivity (32) one cannot be sure that immune responsiveness has been induced by live M.leprae. These cautions may be more relevant to the endemic situation, than the epidemic where cumulative prevalence rates of over 30\% in 6 years have been recorded (33) and it must be presumed that infections normally take, and are controlled immunologically. If immune conversion does equate with the establishment of infection, then the findings of 
Godal and Negassie (10) and Abe (18) are highly significart, as they suggest that in the endemic situation the immune response protects a large proportion of the infected population against the development of disease. How large a proportion I will try to assess shortly.

The other important study is that of Dharmendra and Chatterjee of 1956 (34), who lepromin tested a population living in an endemic area, and followed it for 15 to 20 years. They found that $3.2 \%$ of those originally Mitsuda positive developed leprosy, in contrast with $14.9 \%$ of those (47 individuals) Mitsuda negative. While of those who persisted negative after 3 challenges with lepromin $62.5 \%$ (of 16) developed leprosy, and in eight out of ten cases it was lepromatous. Despite the arguments that surround the interpretation of the lepromin test and thus the meaning of this study, it is difficult to escape the conclusion that those individuals who are capable of mounting a CMI response to one or a few heat resistant antigens of M.lepräe are one fifth as likely to develop leprosy, and almost never develop lepromatous leprosy. The study does not however allow a comparison of the value of natural infection against lepromin testing as a means of inducing protective immunity. Nor does the study help answer the question of whether immune unresponsiveness is genetic or acquired, as Newell has suggested it does ( 35 ) because it must now be assumed that the population was exposed to M.leprae before the lepromin testing was carried out. Here again Godal and Negassie and Abe's results are critical. They show that infection normally takes place in childhood. If this is so, then in endemic areas all adult leprosy and the majority of all leprosy, is post-primary. That is to say it is due either to reinfection, which is in fact occurring all the time is therefore unlikely to be important, or to a breakdown, usually temporary, of acquired resistance. Lurie found it impossible to establish a second tuberculous lesion in a resistant rabbit harbouring a primary lesion, even if the primary later broke down and permitted lethal disseminated disease (25). If this concept is true, then in leprosy the skin is unlikely to be the normal portal of entry. Without accurate incidence figures it is very difficult to estimate how large a proportion of the population are solidly and permanently protected against the development of disease by the immune response to infection. Browne's figures from his paper on self healing leprosy in an endemic area of the Congo (36) are, that over an eight year period, $25 \%$ of the adult population over 20 years of age developed leprosy. If these figures are generally applicable in endemic areas, it becomes apparent that the immune response is inefficient at protecting against the eventual development of leprosy from a subclinical infection. The fact that much adult leprosy is self healing, 44\% in Browne's series, simply masks the situation.

The concept then emerges that all adults in an endemic area have latent leprosy, and the demonstration by Abe that the prevalence of antibodies in adults does not decline, supports this 
view. This latent infection has the capacity to break through, with different frequencies in different communities. In the endemic situation, breakthrough is common

while in the dying endemic state ureakthrough is rare. LTT and serological studies in a southern European country could test this hypothesis.

Browne's figures also show that the incidence of leprosy in adults over the age of 20 years is 2 to 3 times that of children under 10 years, and suggests the development or acquisition of immunosuppressive factors in adult life. By contrast the children are 4 to 8 times more likely to develop nonself healing disease. These two observations cannot both be explained purely immunologically, and suggest the operation of other, possibly genetic factors in childhood, selecting for susceptibility to lepromatous disease.

The answer then to the question, does subclinical infection protect against the subsequent development of leprosy? is : yes but it depends. It protects a large proportion initially, but this protection is not as efficient as it might seem; protection may modulate by genetic factors especially in childhood, by suppressive factors in early adult life, and by unknown factors which alter the pattern of disease from epidemic, to endemic to waning.

Finally, one must consider experimental evidence for protection. There are animal data, that inoculation of an adequate dose of suitably prepared, whole dead M.leprae protects mice against a subsequent foot pad infection with live M.leprae

(37). Vaccination with dead M.leprae has also been shown to protect the more susceptible armadillo (38). The relevance of these data to man must remain uncertain, but it encourages the hope that an appropriate preparation or extract of dead M.leprae may be found which, when inoculated into previously unexposed humans, will induce protective immunity. At Culion it was considered that repeated lepromin testing of very young children conferred some protection against disease and type (27). The fact that ribosomal fractions, phosphatides and cell walls of M.tuberculosis have, in various situations shown to be protective, adds further encouragement. The difficulty with man may be in identifying the unexposed, immunologically unprimed population who are not so young as to be unresponsive.

The mechanism by which subclinical infection protects against the development of clinical disease is unknown. Positive LTT and lepromin tests suggest the presence and efficiency of cellular mechanisms which may contain persisting organisms. It is not known whether the presence of antibody signifies an efficient effector mechanism or simply bacterial persistence. Nor are the mechanisms which induce suppression known. They may be related to the organism itself or to its ability to persist in an immunologically priviledged site such as the Schwann cell (39) or to the host, for example puberty or pregnancy, as has been invoked frequently in individual patients. But the regularity with which the containment of a subclinical infection fails, suggests the operation of a mechanism as yet unidentified. 
3. HEALING OF ESTABLISHED DISEASE : Healing not only prevents the further development of deformity, but also removes a potential reservoir of infection. Most studies of the immune phenomena in leprosy have been conducted in patients with clinical disease, and these results I have summarised. Healing is the only one of the five situations in which a mechanism for the killing of M.leprae has been established. Godal (2) showed that human monocytes were capable of destroying M.leprae in the presence of lymphocytes from tuberculoid patients. In contrast, lymphocytes from lepromatous patients could not confer this ability to monocytes. There has been a tendency to assume that this killing mechanism, loosely equated with CMI, is the protective mechanism at all stages of the infection. But there is no proof that this is so. It is an inefficient process, taking years to complete and accompanied by much hypersensitivity and tissue destruction. Immunity to reinfection (no 4 below) would by contrast seem to be efficient, quick and clean. Conversely, host mechanisms to contain a few dormant bacilli (no 2 above) may not be the same as those needed to control the spreading infection that develops when containment breaks down. Lara reported that the initial lesion in $45 \%$ of children with leprosy at Culion was a bacilliferous papule, which healed in almost all instances (27). Was this because of CMI?

4. PROTECTION AGAINST REINFECTION AFTER RECOVERY FROM DISEASE: Reinfection may be difficult to distinguish from relapse in patients recovered from paucibacillary disease. Relapses are commonest within the first two years of stopping treatment, and rare after four years (40). Disease following reinfection would be expected to appear later. There is little to suggest that reinfection which must be continuous in endemic areas, ever produces disease. If this were true it would support the concept that protection after clinical disease is very much more solid than that accompanying subclinical infection, and presumably involves an added or different mechanism.

5. PROTECTION AGAINST RELAPSE : Relapse of patients after treatment for multibacillary is of great epidemiological importance, but as these patients have never shown evidence of an effective immune response they will not be considered further here. Relapse of selfthealing and treated paucibacillary disease is well known and also of potential epidemiological importance as downgrading may occur and permit the development of multibacillary disease, and so establish a new reservoir of infection. In India the relapse rate for treated tuberculoid patients was over $30 \%$ after two years of dapsone treatment, declining to under $1 \%$ after eight years treatment (40). In Ethiopia 15\% of tuberculoid (TT) patients treated for over $1 \frac{1}{2}$ years relapsed and $15 \%$ of BT treated for over 5 years (41). That such prolonged treatment is necessary to permit complete healing in a disease which exhibits such marked evidence of CMI, suggests the action of a suppressor mechanism, such as Nath found (4). It would be of relevance to know whether this mechanism persists through treatment and recovery. 
Relapses of disease, or the emergence of clinical disease from latent leprosy, late in life may be of epidemiological importance by creating a new and unexpected reservoir of infection in the declining endemic situation. If lepromatous leprosy were to appear in a grandmother, who looked after her grandchildren, and who died before being diagnosed, leprosy would seem to skip two generations and its reappearance in the community or family would be hard to explain. There were several examples of this given during the clinical presentations at Symposium on Leprosy in Europe, St Margheurita, 1981.

In the absence of adequate knowledge of mechanisms of healing and immunity it is not possible to discuss how they fail.

\section{Relative}

What is the relative importance of specific immunity in protecting against leprosy? Relative to what? Relative to a number of unrelated factors each of which may be of different significance according to the stage or state of leprosy in the community. As most of the factors will be dealt with by others, I shall comment briefly only on three of them: genetic susceptibility, other mycobacteria, and intensity of transmission, in order to suggest that the relative importance of immunity varies as to whether the disease in the community is epidemic, endemic or waning.

The argument that previous exposure to one species may preempt the host response to subsequent infection with another species of mycobacteria has been advanced by Stanford and his group $(42,43)$ on experimental grounds. He has extended this argument to the epidemiology of leprosy postulating that wild mycobacteria will affect responses to subsequent infection either with M.leprae or with BCG and will thus affect the pattern of disease and the protective response of BCG vaccination. Lefford's experiments lend some support to this hypothesis (44). He showed that the protective response of BCG against M.lepraemurium in mice could be prevented by previous intravenous injection of M.lepraemurium which suppressed CMI responses to both organisms.

The discussion which follows is intended simply to illustrate possible situations in which these three factors might relate to immunity in creating patterns of disease. Supportive data are scanty. My assumptions for this discussion are l. that infection with 1 leprae causes predominantly subclinical leprosy and smaller numbers of cases of self healing, borderlirie and lepromatous disease, 2. that subclinical infection is responsible for the late appearance of disease of all categories including cases of disease in old age, 3. that borderline disease is the major cause of disability and 4. that lepromatous disease provides the reservoir of infection and thus the maintenance of the disease and its characteristics in the community. 


\section{EPIDEMIC LEPROSY}

The rapid spread of leprosy and its high incidence are striking features of an epidemic. Leiker (45) and other (46) have rehearsed the arguments: Lepromatous cases are few, so intensity of transmission is not especially high, there is no evidence that M.leprae becomes abnormally virulent because self healing minor tuberculoid leprosy is the commonest type. It seems likely that the virgin population is particularly susceptible to infection. This is not surprising as there is no preexisting immunity conferred by subclinical infection, but the possibility of genetic susceptibility arises. If it is present it is to leprosy as a whole, not to lepromatous disease. Lurie noted that genetically determined resistance to tuberculosis in rabbits was independantly directed either against the establishment of the infection or the spread of the infection (25). It is also possible that the decline of epidemic leprosy, as in Europe over a period of 400 years was aided, if not brought about, by the elimination of one susceptible population by deathconfinement or sterility. For genetic reasons subclinicalimmunity is relatively rare and inefficient in an epidemic and herd immunity is not established. Amplification of the disease would be adequate through the relatively small numbers of lepromatous cases and late relapses would be relatively few and too late to be of much importance. In the Nauru epidemic the incidence which peaked at $15 \%$ in the $3 r$ year had declined to under $1 \%$ in the 7 th, 8 th and 9 th years (33).

Guinto and Rodriguez (47) pointed out that the fresh introduction of leprosy into a community did not necessarily lead to an epidemic, even though there were no detectable differences between the susceptible and apparently non susceptible communities. It is possible, that previous exposure to cross reacting mycobacteria might be relevant to such a situation. Leiker suggested (45) that tuberculin positivity conferred some protection against the epidemic spread of leprosy in New Guinea.

\section{ENDEMIC LEPROSY}

High endemicity tends to be associated with dense populations. Dense population is associated with increased contact with lepromatous patients and thus with greater intensity of transmission. In Culion colony 36\% of children kept in contact with infectious mothers for 3 to 6 years developed leprosy before the age of six, with a sharp decrease thereafter, suggesting that intensity of infection advanced the age of onset in susceptibles (27). Contacts qf lepromatous patients have a 2 to 6 fold greater risk of developing leprosy than contacts of tuberculoid patients. Heavy inocula might also cause the larger proportion of borderline cases that are observed in the endemic situation and so produce more disability in the community than occurs in an epidemic. In experimental situations large inocula shorten the incubation period 
and increase the chance of disease in experimental mouse leprosy. If this applies in man, heavy inocula might contribute to the higher rate of leprosy in children compared with the epidemic situation. Identification and treatment of lepromatous patients would be expected to reduce endemicity considerably as the most susceptible genetically would be reduced, and some herd immunity exists due to subclinical infection; but the large numbers of subclinical cases would permit the escape of.new adult lepromatous cases who would keep the endemic going.

Previous exposure of the community to other mycobacteria might be expected to affect the degree of endemicity but the intense transmission of M.leprae would dominate. Results of BCG vaccination in different communities would be very difficult to interpret because previous exposure to wild mycobacteria may have preempted the response to BCG (42) and also because the majority of people being vaccinated would already have been exposed tholeprae. The action of BCG might then be that of an adjuvant and promote healing of subclinical disease and suggest a good degree of protection, it might, on the other hand, induce hypersensitivity and precipitate latent borderline disease which would give the appearance of poor protection (12), or its action might be entirely preempted, and so have no effect. The experimental work of Sehpard (37) and Lefford (44) show good protection by BCG and suggests it acts by inducing specific immunity to M.leprae through shared antigens. Its failure in certain human populations remains a puzzle.

\section{WANING LEPROSY}

The genetically most susceptible pool has disappeared. Intense transmission is necessary to maintain the infection, but overcrowding is becoming less as the community advances. Subclinical cases are few so that specific herd immunity is decreasing. The increasing use and efficient administration of BCG against tuberculosis would provide better protection against leprosy. Exposure to wild mycobacteria may diminish. Several factors, therefore, might be expected to amplify protection. The situation is finally reached when even the reintroduction of M.leprae into the community cannot start a ńew outbreak. Certain individuals, however, are still susceptible to intense transmission. There are no longer enough lepromatous cases to amplify the disease in the community. Nevertheless in the occasional subclinical case the infection escapes containment, perhaps because of nonspecific suppressive factors in old age, and grandmother gets lepromatous leprosy. The other adults of the family many of whom may have been immunised by exposure to their grandmother, do not get leprosy, but the grandchildren get subclinical disease and so there is a potential for the disease to persist in the family. Perhaps a third type of genetic susceptibility characterises the family; there may even be a degree of inbreeding in communities where leprosy has lingered longest ( 35 ). 


\section{Summary \& Conclusions}

The studies of Godal and Negassie and Abe demand a revision of present concepts of the epidemiology of leprosy. Subclinical infection is the rule and provides a weak measure of specific protection, but also provides a latent reservoir of infection in the community. Other forms of specific protection may also exist. The pattern of disease and resistance will be affected by other factors, notably genetic susceptibility, intensity of transmission and exposure to other mycobacteria, both wild and BCG. The relative importance of these factors is different when leprosy is epidemic, endemic or waning.

\section{References}

1 Myrvang B, Godal T, Ridley D S, Froland S S and Song Y K. Immune responsiveness to Mycobacterium leprae and other mycobacterial antigens throughout the clinical and histopathological spectrum of leprosy. Clin exp Immunol,1973,14, 541

2 Godal T, Rees R J W, Lamvik J O. Lymphocyte mediated modification of blood derived macrophage function in vitro : including inhibition of intracellular mycobacteria. Clin exp Immunol, 1971, 8, 625

3 Godal T, Mykelstad B, Samuel D R, Myrvang B. Characterisation of the cellular immune deficit in lepromatous leprosy : a specific lack of circulating Mycobacterium leprae reactive lymphocytes. Clin exp Immunol, 1971, 9, 821-831

4 Nath I, Van Rood J J, Mehrā N K, Vaidya M C. Natural suppressor cells in human leprosy : the role of HLA-D identical peripheral lymphocytes and macrophages in the in vitro modulation of lymphoproliferative responses. Clin. exp Immunol, 1980, 42,203-10

5 Stoner G L. Hypothesis : do phases of immunosuppression during a Mycobacterium leprae infection determine the leprosy spectrum. Lepr Rev, 1981, 52, 1-10

6 Mehra $V$, Mason L H, Fields J P, Bloom B R. Lepromin-induced suppressor cells in patients with leprosy. J Immunol, 1979 $123,1813-1817$

7 Mehra V, Mason L H, Rothman W, Reinherz E, Schlossman S F, Bloom $B R$. Delineation of a human $T$ cell subset responsible for lepromin-induced suppression in leprosy patients. J Immunol, $1980,125,1183-1188$

8 Touw J, Stoner G L, Belehu A. Effect of Mycobacterium leprae on lymphocyte proliferation : suppression of mitogen and antigen responses of human peripheral blood mononuclear cells. Clin exp Immunol, 1980, 41, 397-405

9 Shepard C C, Draper P, Rees R J W, Lowe C. Effect of purification steps on the immunogenicity of Mycobacterium leprae. Br J exp Path, 61, 376-379

10 Godal $T$, Negassie $\bar{K}$. Subclinical infection in leprosy. Brit med $\mathrm{J}, 1973,3,557-559$ 
11 Lloss 0. In vitro lymphocyte response to purified protein derivative, BCG, and Mycobacterium leprae in a population not exposed to leprosy. Infect Immun. 1975, 11, 1163-1169

12 Stoner $\mathrm{S}_{\mathrm{L}} \mathrm{L}$, Nsibambi J, Warndoff J, Belehu A. Borderline tuberculoid leprosy following BCG vaccination : a case report. Int J Leprosy, 1981, 49

13 Harboe M, Closs I. Bjornvatn B, Kronvall G, Axelsen N H. Antibody response in rabbits to immunisation with Mycobacterium

Infect Immun, 1977, 18, 792-805

14 Closs 0 , Reitan L J, Negassie K, Harboe M. Lymphocyte stimulation in vitro with a purified antigen prepared from Mycobacterium leprae. Scand J Immunol, 1979, 10, 363

15 Axelsen N H, Harboe M, Closs 0, Godal T. BCG antibody profiles in tuberculoid and lepromatous leprosy. Infect Immun, 1974, 9, , $952-958$

16 Harboe M, Closs O, Bjune G, Kronvall G, Axelsen N H. Mycobacterium leprae antibodies detected by radio immunoassay. Scand J Immunol, 1978, 7, 111-120

17 Abe M, Izumi S, Saito T, Mathur S K. Early serodiagnosis of leprosy by indirect immunofluorescence. Lepr India, 1976 $48,272-276$

18 Abe M, Yoshino Y, Saikawa K, Saito T. Fluorescent leprosy anti body absorbtion (FLA-ABS) test for detecting subclinical infection of Mycobacterium leprae. Int J Lepr, 1980, 48, 109-119

19 Myrvang B, Feek C M; Godal T. Antimycobacterial antibodies in sera from patients through the clinicopathological spectrum of leprosy. Acta Path Microbiol Scand B, 1974, 82, 701-706

20 Closs 0, Mshana R N, Harboe M. Antigenic analysis of Mycobacterium leprae. Scand J Immunol, 9, 297-302

22 Bryceson A D.M', Mechanisms of disease in leishmaniasis. Symp Brit Soc Parasit 1975, 13, 85-100

23 Brennan P J, Barrow W W. Evidence for species-specific lipid antigens in Mycobacterium leprae. Int J lepr, 1980, 48, 382-387

24 Yoder L, Naafs $\bar{B}$, Harboe M, Bjune G. Antibody activity against Mycobacterium leprae antigen 7 in leprosy. Rev, 1979,50 113-121

25 Lurie M B. Native and acquired resistance to tuberculosis. Amer J Med, 1950, 9, 591-610

26 Poulter LW. Mechanisms of immunity to leishman for a changing basis of protection in self limiting disease. Clin exp Immunol, 1980, 39, 14-26

27 Lara C B, Leprosy in children, general considerations : initial and early stages. WPR/LEP/24, 1961 WHO-GeneVa

28 Melsom R, Duncan M E, Harboe M, Bjune G. Antibodies against Mycobacterium leprae antigen 7 from birth to 18 months of age. Clin exp Immunol, 1980, 42, 107-113

29 Davy T F, A day in the life of Yeeranna - a cautionary tale. Lepr. Rev 1978, 49, 269-274

30 Barnetson R St C, $\overline{B j} u n e ~ G$, Duncan M E. Evidence for a soluble lymphocyte factor in the transplacental transmission of T-cell responses tn leprae, Nature, 1976, 260, 150-151 
31 Bradley D J, Kirkley J. Regulation of leishmania populations within the host. The variable course of leishmania donovani infection in mice. Clin exp Immunol, 1977, 30, 119

32 Doull J A, Guinto R S, Mabalay M C. Effect of BCG vaccination lepromin testing and natural causes in inducing reactivity to lepromin and to tuberculin. Int J Lepr, 1957, 25, 13-33

33 Bray G W. The story of leprosy at Nauru. Proc Roy Soc Med, 1930 23/2, 1371-1374

34 Dharmendra, Chatterjee $K R$. Prognostic value of the lepromin test in contacts of leprosy cases. Int J Lepr 1956, 24, 315-318

35 Newell K. An epidemiologists view of leprosy. Bull.wid. Hlth Org $1966,34,827-857$

36 Browne $\bar{S}$, Self-healing leprosy : report on 2749 patients. Lepr Rev, 1974, 45, 104-111

37 Shepard C C. The immunogenic competence of killed M.leprae. Int J Lepr, 1978, 46, 118-119

38 Ki rchheimer W F. Qauntitative aspects of experimental infection of armadillos with Mycobacterium leprae. In F Latapi et al Eds. Leprosy Amsterdam-Oxford-Princeton : Excerpta Medica, $1980, \frac{27-31}{27}$

39 Stoner G L. Importance of the neural predilection of Mycobacterium leprae in leprosy. Lancet, 1979, 2, 994-996

40 Vellut C, Lechat M F. Miss C B. Tuberćuloid relapses. In Leprosy (op cit ref 38) p 293-298

41 Touw L, Naafs B. Relapses in leprosy after release from control. Lepr Rev, 1979, 50, 123-127

42 shield M J, Stanford J L, Rook G A W. The reason for the reduction of the protective efficacy of BCG in Burma. In Leprosy (op citref 38) p. 8

43 Rooke G A W, Stanford J L, Shield M J. Characterisation in mouse and man of two distinct forms of delayed skin-test response, relevant to mycobacterial infections. In Leprosy (op cit ref 38$)$ P. 158

44 Lefford M J, Morgan R, Logie P S. Effect of Mycobacterium bovis BCG vaccination upon Mycobacterium lepraemurium infections. Infect Immun, 1980, 28, 860-866

45 Leiker DL. Epidemiological and immunological surveys in Netherlands New Guinea. Lep Rev, 1960, 31, 241-259

46 Wade $H$ W, The leprosy epidemic at Nauru. Int J Lepr, 1952,20, 1-28

47 Guinto R S, Rodriguez J N, A leprosy survey of a control area Santander, Cebu, Philippines. Int J Lepr, 1941, 9, 315-325 